\title{
Quality factor and finesse optimization in buried InGaAsP/InP ring resonators
}

\author{
Caterina Ciminelli \\ c.ciminelli@poliba.it
}

Vittorio M. N. Passaro

Francesco Dell'Olio

Mario N. Armenise
Dipartimento di Elettrotecnica ed Elettronica, Politecnico di Bari, Via Re David 200 - 70125 Bari, Italy

Dipartimento di Elettrotecnica ed Elettronica, Politecnico di Bari, Via Re David 200 - 70125 Bari, Italy

Dipartimento di Elettrotecnica ed Elettronica, Politecnico di Bari, Via Re David 200 - 70125 Bari, Italy

Dipartimento di Elettrotecnica ed Elettronica, Politecnico di Bari, Via Re David 200 - 70125 Bari, Italy

Quality factor and finesse of buried $\mathrm{In}_{1-x} \mathrm{Ga}_{x} \mathrm{As}_{y} \mathrm{P}_{1-y} / \mathrm{InP}$ ring resonators have been optimized in this paper by a very general modelling technique. Limiting effect of propagation loss within the ring has been investigated using a three-dimensional (3D) highly accurate complex mode solver based on mode matching method to analyze bending loss dependence on ring radius and wavelength. Coupling between straight input/output ( $1 / 0$ ) bus waveguides and ring resonator has been studied by 3D Beam Propagation Method (BPM), deriving coupling loss and coupling coefficient for a large range of ring radius and bus waveguides-ring distance values (for both polarizations). Ring resonator has been modelled by the transfer-matrix approach, while finesse and quality factor dependence on radius has been estimated for two resonator architectures (including one or two $1 / 0$ bus waveguides) and for quasi-TE and quasi-TM modes. Guiding structure has been optimized to enhance resonator performance. The modelling approach has been validated by comparing results obtained by our algorithm with experimental data reported in literature. Influence of rejection (at resonance wavelength) at through port on quality factor and finesse has been widely discussed. A quality factor larger than $8 \times 10^{5}$ has been predicted for the ring resonator employing only one $1 / 0$ bus waveguide and having a radius of $400 \mu \mathrm{m}$. This resonator exhibits a rejection of $-8 \mathrm{~dB}$ at through port. [D0I: 10.2971/jeos.2009.09032]

Keywords: semiconductor ring resonators, high quality resonators, angular velocity photonic sensors, optical gyros

\section{INTRODUCTION}

Although passive integrated optical ring resonators have been firstly theoretically proposed since about forty years for wavelength filtering [1], only in the last two decades there has been an intense research effort to employ them as building blocks in a large number of photonic applications, such as multiplexing, demultiplexing, filtering, switching, and modulation [2, 3]. Very recently, an eight-channel demultiplexer based on ring resonators fabricated in Silicon-on-Insulator (SOI) technology and to be used in WDM signals processing has been demonstrated [4]. A racetrack shaped ring resonator realized by polymer materials has been employed to fabricate an electrooptic modulator having a bandwidth up to $165 \mathrm{GHz}$ [5].

The use of integrated optical ring resonators for sensing is becoming a more and more attractive research field. A number of passive architectures based on large-radius ring resonators have been proposed and experimentally demonstrated for Sagnac effect-based optical angular rate estimation [6]-[12]. Some ring resonators properly designed to be included in passive integrated optical gyroscopes have been experimentally investigated [13]-[15]. A large-radius ring resonator for gyroscopic applications in which propagation loss is compensated by optical gain induced by a pump signal (at $830 \mathrm{~nm}$ ) has been fabricated in neodymium-doped glass exhibiting a quality factor around $2 \times 10^{7}$ (at $\left.1060 \mathrm{~nm}\right)$ [16].
Chemical and biochemical optical sensing by integrated ring resonators has been widely demonstrated by fabricating (using a wide spectrum of materials as glass, polymers and silicon nitride) highly compact devices capable to detect a propagating mode effective index change around $10^{-6}-10^{-7}$ [17][20]. Finally, micro-displacement and acceleration measurements have been also theoretically and experimentally proved by optical micro-ring resonators [21, 22].

First ring resonators have been fabricated by polymethyl methacrylate film deposited on quartz, silver ion exchange in glass, and silica-on-silicon technology [23]-[25]. To obtain large quality factors (up to $2 \times 10^{7}$ for ring resonator realized in Silica-on-Silicon technology) by these low index contrast technologies, ring resonators with large radii (around $1 \mathrm{~mm}$ ) and consequently reduced free spectral range (FSR) are needed.

In the last decade other materials have been employed for fabrication of ring resonators, such as III-V semiconductors, silicon, silicon nitride, polymers, and lithium niobate [26]. Electro-optic polymers are very attractive materials for large bandwidth ring resonator-based optical modulators, whereas SOI technological platform has permitted to realize ring resonators having a FSR $>30 \mathrm{~nm}$ [27], which are very effective 
for applications related to optical communications. III-V semiconductors technology exhibits the great advantage to allow the monolithic integration of the ring resonator together with an electrically pumped, reliable and high performing laser and other active/passive integrated optical components e. $\mathrm{g}$. semiconductor optical amplifiers (SOAs) [28]-[30].

In ring resonators realized on III-V semiconductors [31]-[35], deeply etched (etch depth around 1-2 $\mu \mathrm{m}$ ) and high index contrast waveguides are usually adopted to maintain ring radius $<10 \mu \mathrm{m}$ and obtain FSR values $>10 \mathrm{~nm}$. The main drawback of this design strategy is the quite limited quality factor and finesse of these devices. Among III-V semiconductor materials, $\mathrm{In}_{1-x} \mathrm{Ga}_{x} \mathrm{As}_{y} \mathrm{P}_{1-y} / \mathrm{InP}$ material system has permitted to fabricate ring resonators having a lager quality factor with respect to those realized using GaAs / $\mathrm{Al}_{x} \mathrm{Ga}_{1-x}$ As material system [36].

An innovative approach for $\mathrm{In}_{1-x} \mathrm{Ga}_{x} \mathrm{As}_{y} \mathrm{P}_{1-y} / \mathrm{InP}$ ring resonators design has been proposed in [35], where a low index contrast waveguide has been employed and so a quite large ring radius $(200 \mu \mathrm{m})$ is needed to avoid too large bending loss. Fabricated resonator has a finesse, a quality factor and a FSR equal to $39,1.13 \times 10^{5}$ and $0.54 \mathrm{~nm}$, respectively. Main geometrical features and performance parameters of III-V semiconductors ring resonators, as reported in literature, are summarized in Table 1.

In this paper we develop an optimization procedure to enhance fully-buried $\mathrm{In}_{1-x} \mathrm{Ga}_{x} \mathrm{As}_{y} \mathrm{P}_{1-y} / \mathrm{InP}$ ring resonator performance. Optical coupling (and related loss) between bus waveguides and resonator has been accurately studied by 3D BPM and propagation loss has been calculated as depending on ring radius and wavelength. Quality factor and finesse dependence on radius has been examined and FSR estimated. Waveguide dimensions and index contrast have been optimized to further enhance the resonator performance. Operating wavelength $\lambda$ is equal to $1.55 \mu \mathrm{m}$.

In Figure 1(a) the cross-section of the investigated waveguide is depicted. $\operatorname{In}_{0.75} \mathrm{Ga}_{0.25} \mathrm{As}_{0.55} \mathrm{P}_{0.45}$ (wavelength band-gap of $1.25 \mu \mathrm{m}$ and refractive index at operating wavelength $n_{1}=$ 3.361) has been considered for the core region. This material is assumed to be deposited on a $\operatorname{InP}\left(n_{2}=3.168\right)$ substrate and be completely buried in $\operatorname{InP}\left(\operatorname{In}_{0.75} \mathrm{Ga}_{0.25} \mathrm{As}_{0.55} \mathrm{P}_{0.45}\right.$ and InP lattice constants are matched). As in [35], the height $(h)$ and the width $(w)$ of buried core region have been fixed as equal to $400 \mathrm{~nm}$ and $900 \mathrm{~nm}$, respectively. This waveguide supports both fundamental quasi-TE and quasi-TM modes. Two ring resonator architectures have been considered. One employing only one I/O bus waveguide (Figure 1(b)) and the other employing two I/O bus waveguides (Figure 1(c)). This last geometry has been assumed as symmetrical, being the two I/O bus waveguides as placed at the same distance from the ring resonator.

\section{PROPAGATION LOSS}

In semiconductor waveguides, loss sources may be due to material absorption or possible geometrical or physical discon-

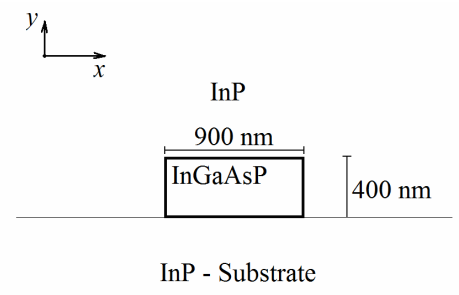

(a)

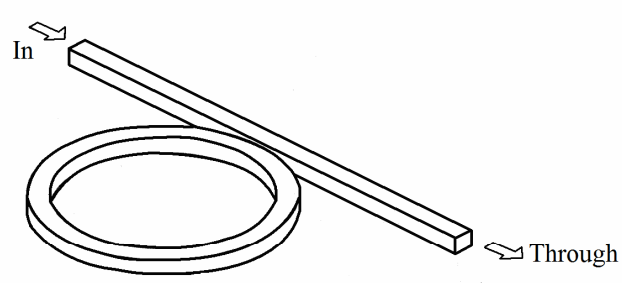

(b)

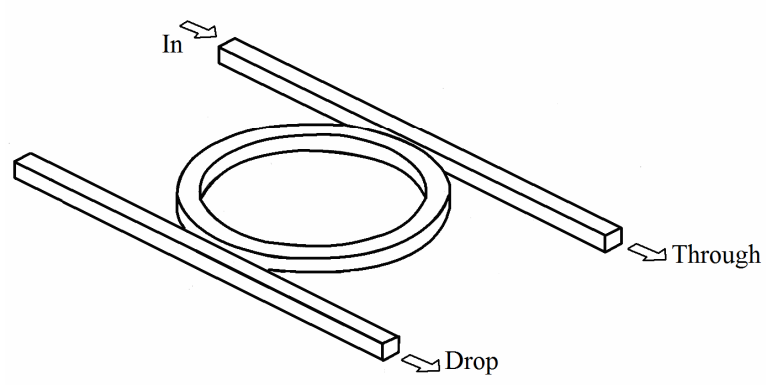

(c)

FIG. 1 (a) Cross-section of the guiding structure used for the ring resonator and $1 / 0$ bus waveguides. (b) Ring resonator architectures employing one and (c) two bus waveguides.

tinuities, which produce some radiated power. Bending induced radiation loss has to be also considered in bent waveguides.

Potential sources of absorption loss in semiconductor waveguides are interband absorption and free carrier absorption (FCA). Interband absorption occurs when photons with energy larger than bandgap are absorbed to excite electrons from the valence band to the conduction band. Therefore, if operating wavelength is longer than the absorption edge wavelength of waveguide materials, interband absorption is avoided. Since operating wavelength (equal to $1.55 \mu \mathrm{m}$ ) is sufficiently longer than InP and In $0.75 \mathrm{Ga}_{0.25} \mathrm{As}_{0.55} \mathrm{P}_{0.45}$ absorption edge wavelengths $\left(\operatorname{In}_{0} \cdot{ }_{75} \mathrm{Ga}_{0.25} \mathrm{As}_{0.55} \mathrm{P}_{0.45}\right.$ and $\mathrm{InP}$ absorption edge wavelengths are equal to $1.25 \mu \mathrm{m}$ and $0.92 \mu \mathrm{m}$, respectively), interband absorption can be neglected in our investigation.

FCA is due to carrier transitions within conduction and valence bands. As the doping concentration increases, the number of free carriers increases and so free carrier absorption increases, too.

For a n-type doped semiconductor, light passing through the crystal is absorbed by an atom and excites an elec- 


\begin{tabular}{|l|c|c|c|c|c|c|c|}
\hline Authors & $\begin{array}{c}\text { 1 BUS / 2 } \\
\text { BUS WGs }\end{array}$ & $\begin{array}{c}\text { Cavity } \\
\text { Length } \\
(\mathrm{mm})\end{array}$ & $\begin{array}{c}\text { Coupling } \\
\text { (Vertical/ } \\
\text { Lateral) }\end{array}$ & $\begin{array}{l}\text { Quality } \\
\text { Factor }\end{array}$ & Finesse & $\begin{array}{c}\text { FSR } \\
(\mathrm{nm})\end{array}$ & $\begin{array}{l}\text { Adopted Materi- } \\
\text { als }\end{array}$ \\
\hline D. Rafizadeh et al. (1998) [31] & 2 & 0.033 & $\mathrm{~V}$ & 1,500 & 20 & 20 & $\mathrm{GaAs} / \mathrm{AlGaAs}$ \\
\hline P. P. Absil et al. (2000) [32] & 2 & 0.051 & $\mathrm{~L}$ & 1,200 & 14 & 18 & $\mathrm{GaAs} / \mathrm{AlGaAs}$ \\
\hline P. P. Absil et al. (2000) [32] & 2 & 0.063 & $\mathrm{~V}$ & 7,040 & 47 & 10.4 & $\mathrm{GaAs} / \mathrm{AlGaAs}$ \\
\hline D. G. Rabus et al. (2001) [33] & 1 & 1.256 & $\mathrm{~L}$ & 19,400 & 5 & 0.4 & $\mathrm{GaInAsP} / \mathrm{InP}$ \\
\hline R. Grover et al. (2003) [34] & 2 & 0.082 & $\mathrm{~L}$ & 6,250 & 32 & 8 & $\mathrm{GaInAsP} / \mathrm{InP}$ \\
\hline R. Grover et al. (2003) [34] & 2 & 0.029 & $\mathrm{~V}$ & 6,200 & 100 & 23 & $\mathrm{GaInAsP} / \mathrm{InP}$ \\
\hline S. J. Choi et al. (2004) [35] & 2 & 1.256 & $\mathrm{~L}$ & 113,000 & 39 & 0.54 & $\mathrm{GaInAsP} / \mathrm{InP}$ \\
\hline
\end{tabular}

TABLE 1 III-V semiconductors passive ring resonators main geometrical features and performance parameters.

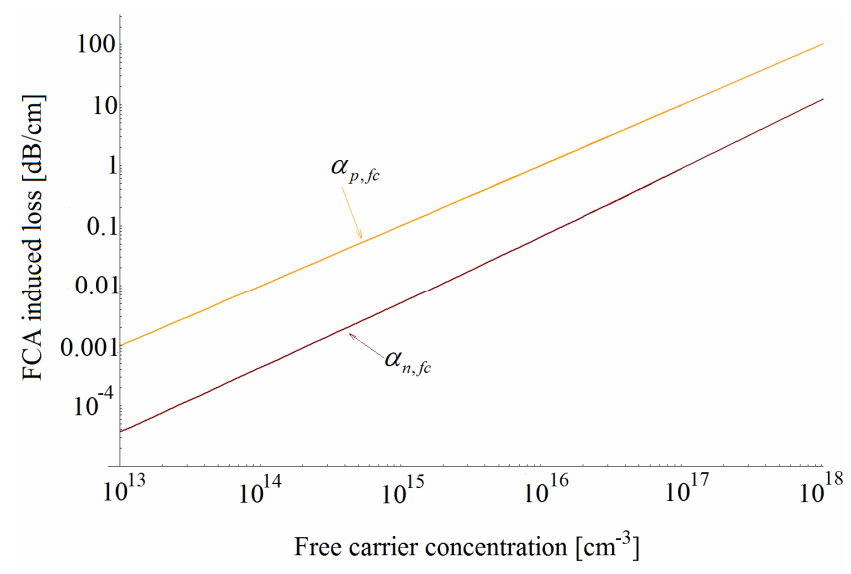

FIG. 2 FCA induced loss dependence on free carrier concentration for n-type and p-type doped $\mathrm{In}_{1-x} \mathrm{Ga}_{x} \mathrm{As}_{y} \mathrm{P}_{1-y}(\log -\log \mathrm{scale})$.

tron at the bottom of the conduction band to a higher energy level. The absorption coefficient $\alpha_{n, f_{c}}$ for $\mathrm{n}$-doped $\mathrm{In}_{1-x} \mathrm{Ga}_{x} \mathrm{As}_{y} \mathrm{P}_{1-y}$ is given by the following equation (at operating wavelength) [37]:

$$
\alpha_{n, \mathrm{fc}}=\frac{4.34 \times 10^{-19} N_{e}}{0.342-0.0172 \log _{10} N_{e}}[\mathrm{~dB} / \mathrm{cm}]
$$

where $N_{e}$ is the electron concentration in $\mathrm{cm}^{-3}$.

For a p-type $\mathrm{In}_{1-x} \mathrm{Ga}_{x} \mathrm{As}_{y} \mathrm{P}_{1-y}, \mathrm{FCA}$ is due to intra-valenceband absorption. In this process, the energy of the absorbed light can excite various transitions between the heavy-hole and the light-hole valence band. The absorption coefficient $\alpha_{p, f_{c}}$ (at operating wavelength) for p-doped $\operatorname{In}_{1-x} \mathrm{Ga}_{x} \mathrm{As}_{y} \mathrm{P}_{1-y}$ is given by [37]:

$$
\alpha_{p, \mathrm{fc}}=9.9 \times 10^{-17} N_{h}[\mathrm{~dB} / \mathrm{cm}]
$$

where $N_{h}$ is the hole concentration in $\mathrm{cm}^{-3}$.

Absorption coefficients $\alpha_{n, f_{c}}$ and $\alpha_{p, f_{c}}$ dependence on free carrier concentration is shown in Figure 2, for p-type and $\mathrm{n}$-type doping. FCA is larger for $\mathrm{p}$-type doping than for $\mathrm{n}$ type one and, as previously anticipated, it increases when free carrier concentration increases. If $\mathrm{InP}$ and $\mathrm{In}_{1-x} \mathrm{Ga}_{x} \mathrm{As}_{y} \mathrm{P}_{1-y}$ are intentionally undoped, free carrier absorption can be usually neglected (InP intrinsic carrier concentration is equal to $\left.1 \times 10^{7} \mathrm{~cm}^{-3}\right)$. On the other hand, if $\operatorname{In}_{1-x} \mathrm{Ga}_{x} \mathrm{As}_{y} \mathrm{P}_{1-y}$ or InP free carrier concentration is larger than $10^{15} \mathrm{~cm}^{-3}$, free carrier absorption cannot be neglected. We have assumed that InP and $\mathrm{In}_{0.75} \mathrm{Ga}_{0.25} \mathrm{As}_{0.55} \mathrm{P}_{0.45}$ are not intentionally doped and, then, we have neglected FCA induced loss.

Bending loss has been calculated by a full-vectorial 3D mode solver based on mode matching method [38] (in computation of optical modes by 3D mode solver, we have assumed a calculation mesh including 80,000 points). This method has been already adopted to investigate anti-resonant reflecting optical bent waveguides in $\mathrm{In}_{1-x} \mathrm{Ga}_{x} \mathrm{As}_{y} \mathrm{P}_{1-y} / \mathrm{InP}$ technology [39] and SOI bent rib waveguides [40]. A perfectly matched layer (PML) located at the outer side of the bend has been employed in our simulations (as in $[39,40]$ ) to absorb the power leaked out of the bend, avoiding any parasitic reflection.

Bending loss dependence on curvature radius $(R)$ has been investigated for $R$ ranging from $40 \mu \mathrm{m}$ to $1000 \mu \mathrm{m}, \lambda=$ $1550 \mathrm{~nm}$, and for quasi-TE and quasi-TM modes (see Figure 3(a)). Bending loss exponentially decreases by increasing $R$ and it is larger for quasi-TM than for quasi-TE mode. This is due to the fact that quasi-TE mode is more confined in the waveguide core region than quasi-TM one (for example, assuming $R=200 \mu \mathrm{m}$, confinement factor in the core region is equal to $42.8 \%$ for quasi-TE and $38.4 \%$ for quasi-TM mode).

Bending loss practically becomes negligible for $R>500 \mu \mathrm{m}$ $\left(\alpha_{b}<10^{-3} \mathrm{~dB} / \mathrm{cm}\right)$ and so it has not been plotted for $R>$ $600 \mu \mathrm{m}$. For $R=200 \mu \mathrm{m}$ (radius value used in [35]) and $\lambda=$ $1550 \mathrm{~nm}$, bending loss is equal to $3.13 \mathrm{~dB} / \mathrm{cm}$ for quasi-TM and $1.45 \mathrm{~dB} / \mathrm{cm}$ for quasi-TE mode. For $R<100 \mu \mathrm{m}$, bending loss is larger than $50 \mathrm{~dB} / \mathrm{cm}$ so, adopting this waveguide technology, we expect that ring resonator having $R<100 \mu \mathrm{m}$ exhibits only modest performance. Consequently the buried waveguide considered in this paper is not employable to realize optical micro-ring resonators.

Assuming $R=200 \mu \mathrm{m}$, wavelength influence on bending loss has been investigated, for both polarizations, varying $\lambda$ in the range from 1500 to $1600 \mathrm{~nm}$ (see Figure 3(b)). Wavelength influence on $\mathrm{InP}$ and $\mathrm{In}_{0.75} \mathrm{Ga}_{0.25} \mathrm{As}_{0.55} \mathrm{P}_{0.45}$ refractive index has been taken into account by the model reported in [37]. Bending loss significantly increases with $\lambda$ for either quasi-TE or quasi-TM mode. For all considered wavelengths, bending loss is larger for quasi-TM than for quasi-TE mode. A similar bending loss dependence on wavelength has been observed (also experimentally) for a Si-wire waveguide [41]. 


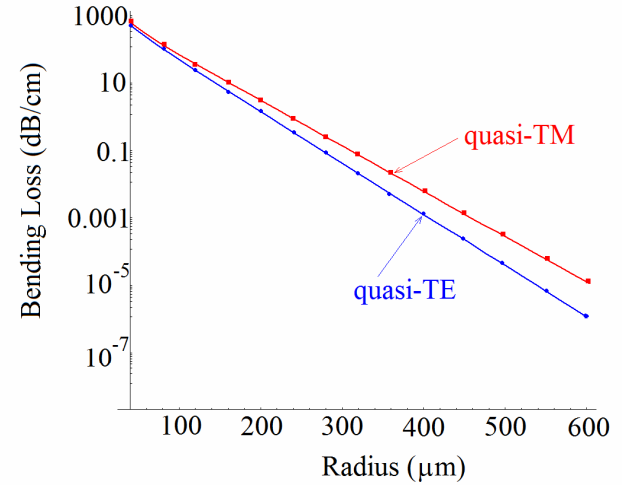

(a)

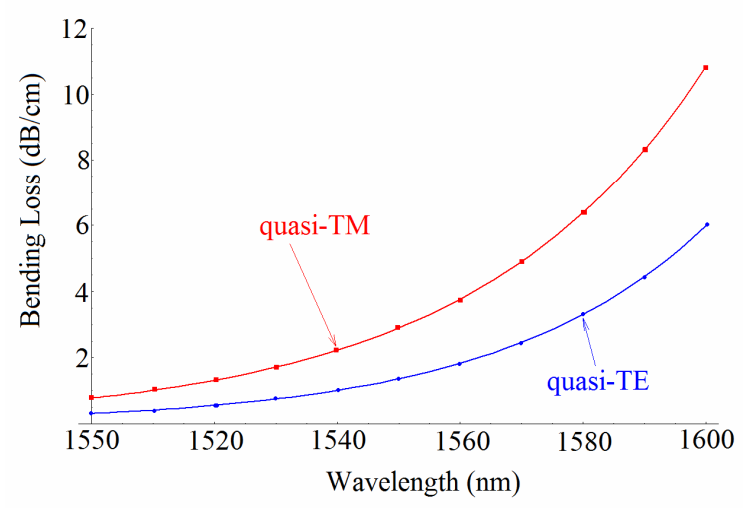

(b)

FIC. 3 Bending loss dependence on radius assuming $\lambda=1550 \mathrm{~nm}$ (a) and wavelength assuming $R=200 \mu \mathrm{m}$ (b). In the plot of bending loss dependence on radius semi-log scale has been used.

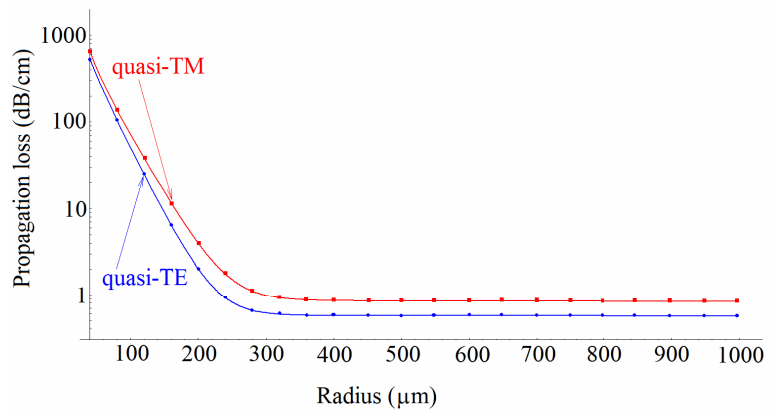

(a)

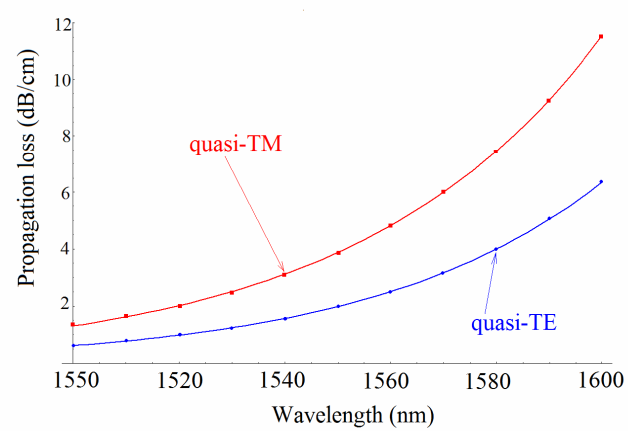

(b)

FIG. 4 (a) Total loss of optical signal propagating within the ring resonator as a function of $R$, for quasi-TE and quasi-TM modes $(\lambda=1550 \mathrm{~nm})$. Semi-log scale has been used. (b) Propagation loss dependence on wavelength for $R=200 \mu \mathrm{m}$ and for both polarizations.
Propagation loss in the ring resonator that we are investigating in this paper is the sum of absorption, bending and scattering losses suffered by the employed $400 \mathrm{~nm}$ high and $900 \mathrm{~nm}$ width buried $\mathrm{In}_{0.75} \mathrm{Ga}_{0.25} \mathrm{As}_{0.55} \mathrm{P}_{0.45} / \mathrm{InP}$ waveguide. Absorption loss has been proved to be negligible, bending loss has been plotted in Figure 3 as dependent on radius of curvature and wavelength, finally, scattering loss has been calculated as a function of radius and wavelength in [42]. Then, assuming $\lambda=1550 \mathrm{~nm}$, optical propagation loss within the ring resonator has been calculated for $R$ ranging from $40 \mu \mathrm{m}$ to $1000 \mu \mathrm{m}$ and for both polarizations (see Figure 4(a)). In this calculation, some results included in [42] has been used. For $R$ values lower than $180 \mu \mathrm{m}$, bending loss is the dominant contribution (bending loss depends exponentially on $R$ ) whereas, for $R$ larger than $300 \mu \mathrm{m}$ scattering loss becomes dominant. Propagation loss decreases by increasing $R$ until it reaches the values of $0.57 \mathrm{~dB} / \mathrm{cm}$ (for quasi-TE mode) and $0.85 \mathrm{~dB} / \mathrm{cm}$ (for quasi-TM mode), representing the minimum propagation loss achievable by this technology (for $\lambda=1550 \mathrm{~nm}$ ). For $R=200 \mu \mathrm{m}$, total propagation loss dependence on wavelength (Figure 4(b)) is dominated by bending loss, so total propagation loss increases by increasing $\lambda$. Propagation loss relevant to quasi-TM mode remains larger than propagation loss relevant to quasi-TE mode in the whole considered wavelength range. In the following sections, operating wavelength has been always fixed as $1550 \mathrm{~nm}$.

\section{COUPLING BETWEEN BUS WAVEGUIDES AND RING RESONATOR}

The optical coupling between I/O straight bus waveguides and the ring resonator has been investigated by 3D BPM [43]. In particular, the structure shown in Figure 5 has been simulated for $R$ ranging from $100 \mu \mathrm{m}$ to $1000 \mu \mathrm{m}$. We have indicated minimal gap between the waveguides (achieved in the middle of the structure) as $g$ ( $g$ is the distance between waveguides sidewalls, as in Figure 5) and structure length has been fixed so that, at the input and output cross-sections, the distance between the waveguides is equal to $10 \mathrm{~g}$ (at this distance the waveguides can be considered uncoupled).

An input optical mode (having amplitude equal to $a$ ) is launched in the straight waveguide and optical mode amplitudes at output cross section have been observed. Propagation step has been varied for all $R$ considered values and it has been fixed as equal to $R \times 10^{-5}$ (for example, for $R=500 \mu \mathrm{m}$ and $g=1 \mu \mathrm{m}$, the propagation step is equal to $5 \mathrm{~nm}$ and the number of propagation steps is around 38,000). Optical

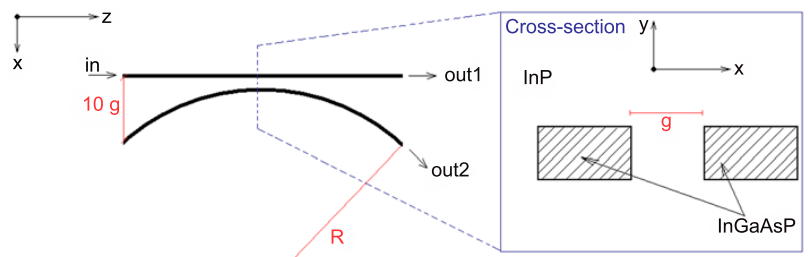

FIG. 5 Structure simulated by 3D BPM to investigate optical coupling between straight $\mathrm{I} / 0$ waveguides and the ring. 


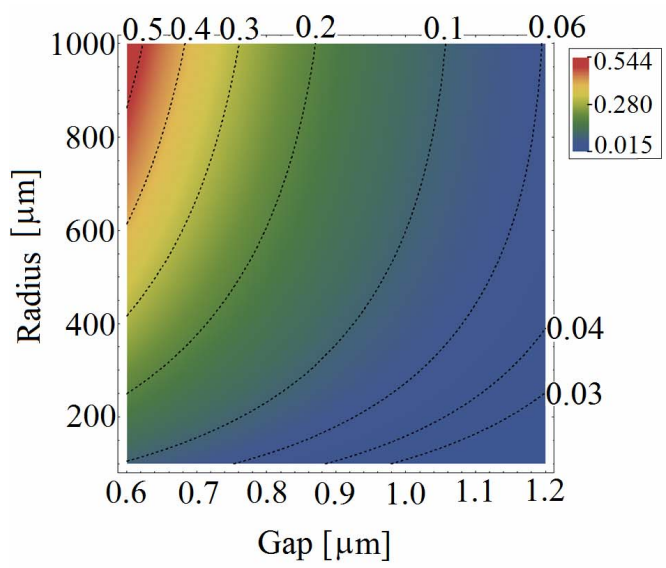

(a)

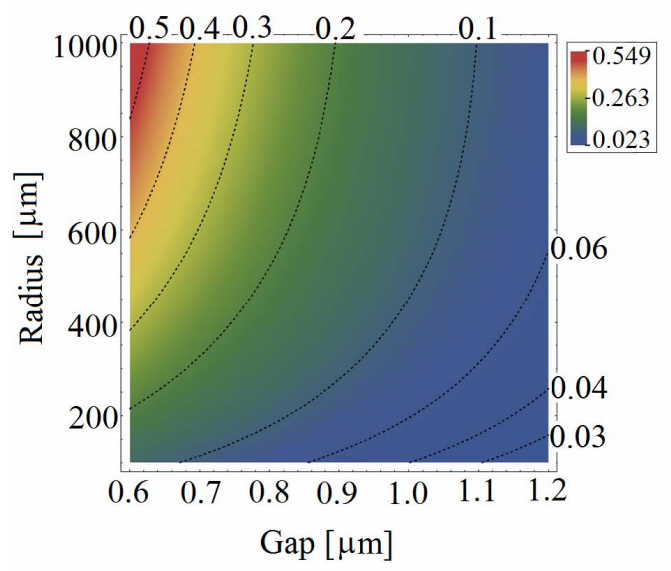

(b)

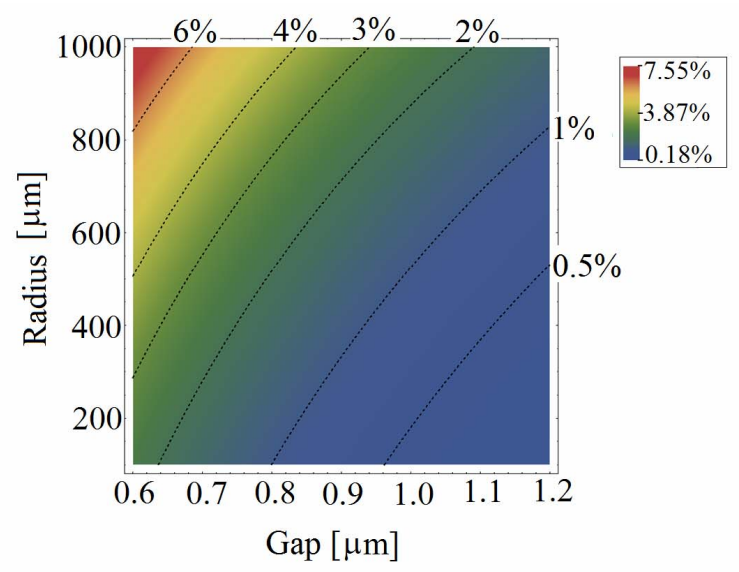

(c)

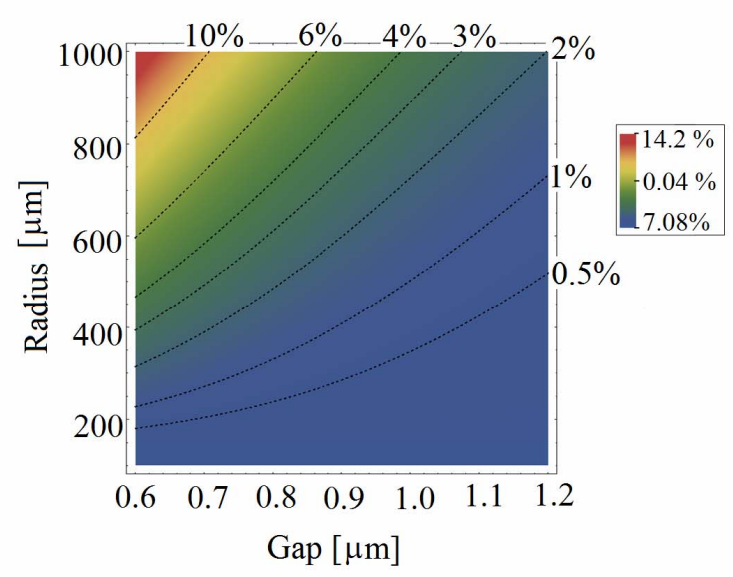

(d)

FIG. 6 Power transfer $\left(\kappa^{2}\right)$ between straight and bent waveguides dependence on $R$ and $g$, (a) for quasi-TE and (b) uasi-TM mode. Coupling loss ( $A_{C}$ ) dependence on $R$ and $g$, (c) for quasi-TE and (d) quasi-TM mode.

mode amplitude at straight waveguide output is indicated as $b$ and optical mode amplitude at the output of bent waveguide is indicated as $c$. The coupling coefficient $\kappa$ between the two modes propagating in straight and bent waveguides is given by the ratio $c / a\left(\kappa^{2}\right.$ indicates the power transfer between the two waveguides), whereas coupling loss $A_{C}$ (usually expressed in percentage) is equal to

$$
A_{C}=1-\frac{b^{2}+c^{2}}{a^{2}} .
$$

The coefficient $\gamma$ (that will be used in the following section), taking into account loss due to the bus waveguides-ring coupling, is defined as equal to $\left(b^{2}+c^{2}\right) / a^{2}$ and so $A_{C}=1-\gamma$.

Power transfer between the two waveguides $\left(\kappa^{2}\right)$ and coupling loss $A_{C}$ (in percentage) have been plotted (in Figure 6) for both polarizations, for $R$ ranging between $100 \mu \mathrm{m}$ and $1000 \mu \mathrm{m}$, and gap $g$ varying between $0.6 \mu \mathrm{m}$ and $1.2 \mu \mathrm{m}$. The dotted curves in the density plots reported in Figure 6 show level curves. Data reported in Figure 6 show that, by decreasing $g$, a power transfer increase is induced and that a $R$ increase produces a power transfer increase (these considerations are valid for both polarizations). Coupling loss exhibits a dependence on $R$ and $g$ quite similar with that exhibited by $\kappa^{2}$, reaching the largest values for largest $R$ value and lowest gap value. For better comparison of coupling loss and power transfer related to different polarizations, we have plotted $\kappa_{T M}^{2}-\kappa_{T E}^{2}$ and $A_{C}^{T M}-A_{C}^{T E}$ (see Figure 7). Figure 7(a) shows that power transfer is larger for quasi-TM mode than for quasi TE one (the difference $\kappa_{T M}^{2}-\kappa_{T E}^{2}$ is always positive), and that the difference between $\kappa_{T M}^{2}$ and $\kappa_{T E}^{2}$ is lower than $2.6 \%$. This difference is maximum for $R=100 \mu \mathrm{m}$ and $g=1.2 \mu \mathrm{m}$ and for $R=1000 \mu \mathrm{m}$ and $g=0.6 \mu \mathrm{m}$. As shown by Figure $7(\mathrm{~b})$, coupling loss related to TM mode is larger than coupling loss related to TE mode for $R>450-470 \mu \mathrm{m}$ (the level curve relevant to $0 \%$ is quite horizontal). The difference $A_{C}^{T M}-A_{C}^{T E}$ reaches its maximum value $(6.65 \%)$ for $R=1000 \mu \mathrm{m}$ and $g=0.6 \mu \mathrm{m}$, and its minimum value $(-2.63 \%)$ for $R=100 \mu \mathrm{m}$ and $g=0.6 \mu \mathrm{m}$.

\section{RING RESONATOR MODELLING AND OPTIMIZATION}

Ring resonator has been modelled by using the standard transfer-matrix approach firstly reported in [44] and the following spectral responses at through port (see Figure 1(b), (c)) have been obtained in case of one or two I/O bus waveguides, 


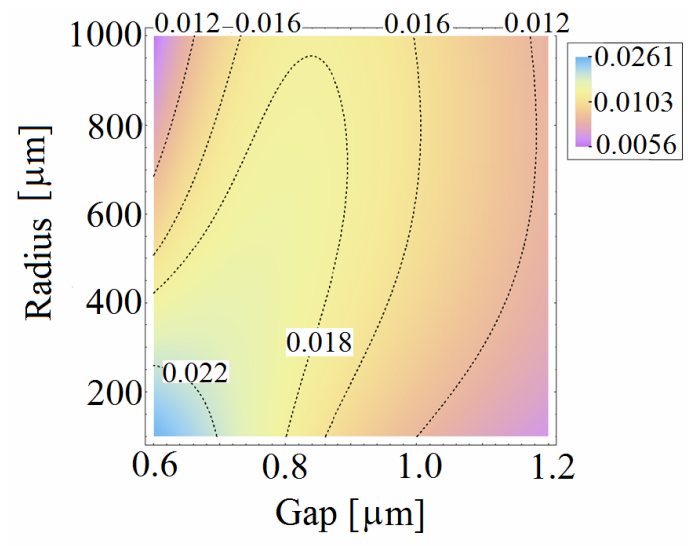

(a)

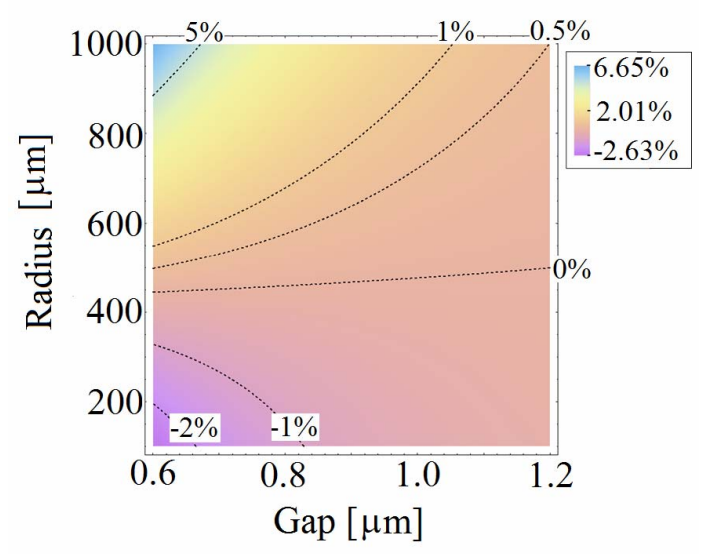

(b)

FIC. 7 (a) Density the plot of $\kappa_{T M}^{2}-\kappa_{T E}^{2}$ and (b) $A_{C}^{T M}-A_{C}^{T E}$.

respectively:

$$
\begin{aligned}
T_{1 b u s W G}(\lambda) & =\left|\frac{\sqrt{\gamma-\kappa^{2}}-\gamma e^{j \beta L} e^{-\alpha L / 2}}{1-\sqrt{\gamma-\kappa^{2}} e^{j \beta L} e^{-\alpha L / 2}}\right|^{2} T_{2 b u s W G s}(\lambda) \\
& =\left(\gamma-\kappa^{2}\right)\left|\frac{1-\gamma e^{j \beta L} e^{-\alpha L / 2}}{1-\left(\gamma-\kappa^{2}\right) e^{j \beta L} e^{-\alpha L / 2}}\right|^{2}
\end{aligned}
$$

where $\alpha$ is the attenuation coefficient (in power) due to propagation loss within the ring (plotted in Figure 4(a) as dependent on $R$ ), $\beta$ is the propagation constant of optical modes within the ring and $L=2 \pi R$ is the resonator length.

$T(\lambda)$ is minimum (equal to $T_{\text {min }}$ ) for $\beta L=2 m \pi$ whereas $T(\lambda)$ is maximum (equal to $T_{\text {max }}$ ) for $\beta L=(2 m+1) \pi$, being $m$ a natural number. The ratio between $T_{\min }$ and $T_{\max }$ has been indicated as through rejection $\left(T_{r j}\right)$ and it is equal to (for the two considered architectures):

$$
\begin{gathered}
T_{r j, 1 b u s W G}=\left|\frac{\left(\frac{\sqrt{\gamma-\kappa^{2}}-\gamma e^{-\alpha L / 2}}{1-\sqrt{\gamma-\kappa^{2}} e^{-\alpha L / 2}}\right)}{\left(\frac{\sqrt{\gamma-\kappa^{2}}+\gamma e^{-\alpha L / 2}}{1+\sqrt{\gamma-\kappa^{2}} e^{-\alpha L / 2}}\right)}\right|^{2} \\
T_{r j, 2 b u s W G s}=\left|\frac{\left(\frac{1-\gamma e^{-\alpha L / 2}}{1-\left(\gamma-\kappa^{2}\right) e^{-\alpha L / 2}}\right)}{\left(\frac{1+\gamma e^{j \beta L} e^{-\alpha L / 2}}{1+\left(\gamma-\kappa^{2}\right) e^{-\alpha L / 2}}\right)}\right|^{2}
\end{gathered}
$$

Starting from the spectral response at through port, some well-known ring resonator figures of merit can be defined.
FSR is the difference between resonance wavelengths (resonance occurs for $\beta L=2 m \pi$ ), the finesse $F$ and the quality factor $Q$ can be defined as:

$$
F=\frac{F S R}{\delta \lambda} Q=\frac{\lambda_{0}}{\delta \lambda}
$$

where $\delta \lambda$ is the resonance spectral width calculated at $T_{\max } / 2$ (usually named Full Wave at Half Maximum, FWHM) and $\lambda_{0}$ is the resonance wavelength.

We have observed that fixing $R$ and incoming light polarization in $T_{r j}$ expressions (reported in Eq. (5)) the only unknown variable is $g$ ( $\kappa$ and $\gamma$ depend only on $g$ if $R$ and polarization are known). So for both ring resonator architectures, for both polarizations and each $R$ value we have calculated by Eq. (5) the $g$ value permitting to obtaining a specified through rejection value $(-8 \mathrm{~dB},-12 \mathrm{~dB}$ and $-16 \mathrm{~dB})$. Obtained $g$ values have been plotted in Figure 8 . In all cases, $g$ value permitting to fulfil the specification imposed on $T_{r j}$ increases for $R<340 \mu \mathrm{m}$ and becomes maximum for $R=340 \mu \mathrm{m}$. Then, for $R>340 \mu \mathrm{m} g$ value linearly decreases. Gap values increase when $T_{r j}$ decreases (in magnitude) and they are larger for quasi-TE than for quasi-TM mode. For $R=200 \mu \mathrm{m}$, $T_{r j}=-8 \mathrm{~dB}$ and the two bus waveguides architecture, we obtain $g=0.9608 \mu \mathrm{m}$ (being lower than $1.5 \%$ the relative error with respect to experimentally reported value, $0.95 \mu \mathrm{m}$ [35]).

Fixing $R, T_{r j}$ and input signal polarization, for both architectures (one/two bus waveguides) we have obtained an unique $g$ value. For every couple of $R$ and $g$ values, we have calculated $\kappa$ and $\gamma$ (see Figure 6). Moreover, for every $R$ value we have estimated the propagation loss $\alpha$ (see Figure 4(a)) and calculated $\beta$ using the 3D mode solver for bent waveguides [38]. At this stage we are able to calculate the spectral response at through port by Eq. (4). After that, F, $Q$, and FSR can be evaluated. Then, we have investigated $Q, F$ and FSR dependence on radius for one/two bus waveguides architectures and both polarizations (see Figure 9 and Figure 10). From Figure 9 it can be observed that high $Q$ (or $F$ ) and high through rejection (in magnitude) are conflicting requirements. Fixing radius, incoming light polarization and architecture, if a large $T_{r j}$ value (for example, $-16 \mathrm{~dB}$ ) is required, we have to accept a lower $Q$ value with respect to the case in which a lower $T_{r j}$ value (for example, $-8 \mathrm{~dB}$ ) is fixed. For a certain $T_{r j}$ value, $Q$ and $F$ dependence on radius is not monotone. $Q$ and $F$ are maximum for $R$ around $300-400 \mu \mathrm{m}$. $R$ values relevant to $Q$ maxima are larger than $R$ values relevant to $F$ maxima. For low $R$ values, as $R$ increases $Q$ and $F$ quickly increase, too. After the maximum, $Q$ slowly decreases when $R$ increases whereas $F$ decrease is faster. Quality factor and finesse are larger for one bus waveguide architecture than for two bus waveguides architecture. Finally, quasi-TE polarization permits to achieve larger $Q$ and $F$ values than quasi-TM one (in fact, quasi-TM mode loss is larger than quasi-TE mode one).

For $R=200 \mu \mathrm{m}$, quasi-TE mode, two bus waveguides architecture and $T_{r j}=-8 \mathrm{~dB}$ (these assumptions are the same as in [35]) we have obtained $F=39.86$ and $Q=1.15 \times 10^{5}$. These values are in good agreement (relative error $<3 \%$ ) with those experimentally estimated [35] and summarized in Table 1. 


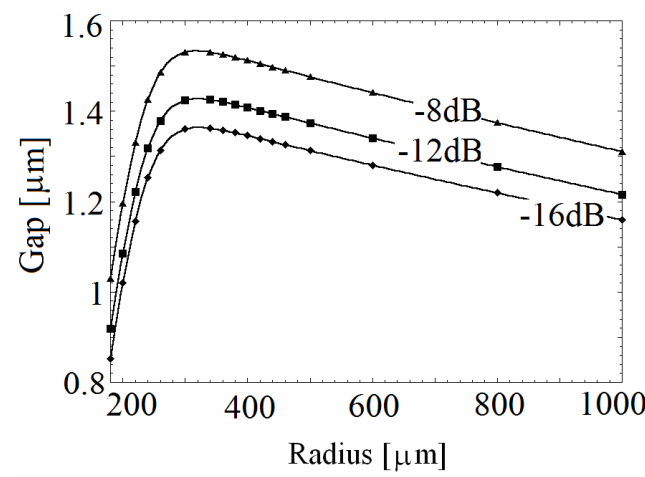

(a)

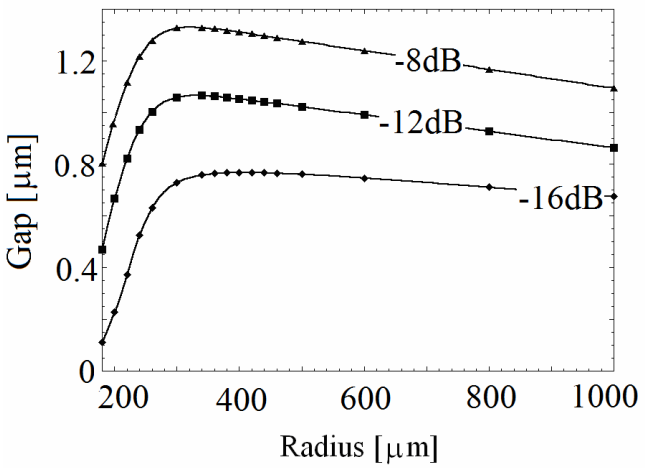

(c)

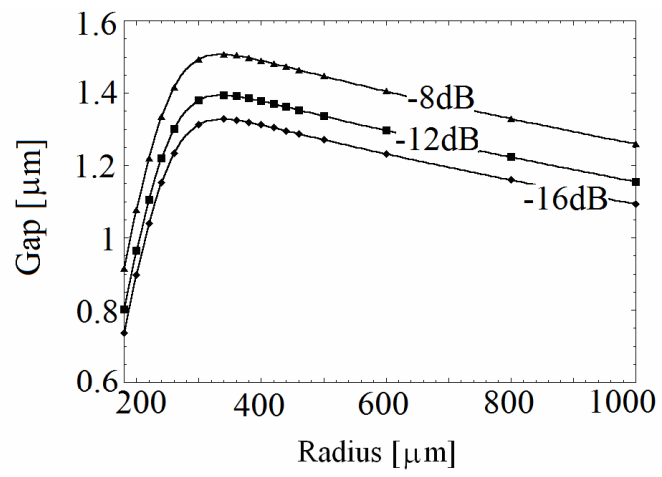

(b)

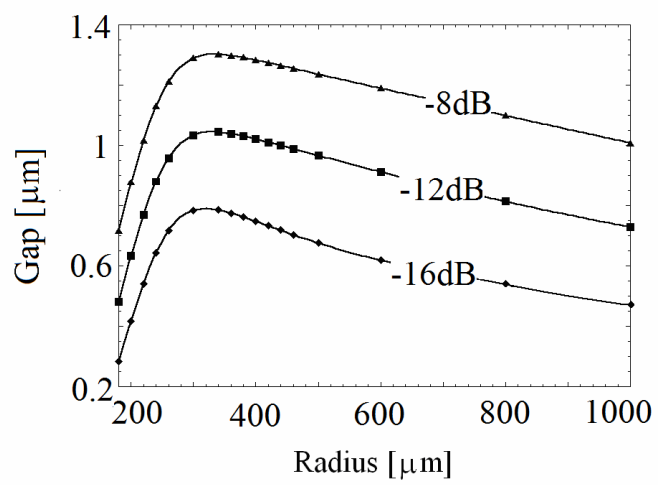

(d)

FIG. 8 (a) Gap value enabling to fulfil the specification on $T_{r j}(=-8 \mathrm{~dB},-12 \mathrm{~dB}$ and $-16 \mathrm{~dB}$ ) for one bus waveguide architecture and quasi-TE mode, (b) one bus waveguide architecture and quasi-TM mode, (c) two bus waveguides architecture and quasi-TE mode, (d) two bus waveguides architecture and quasi-TM mode.

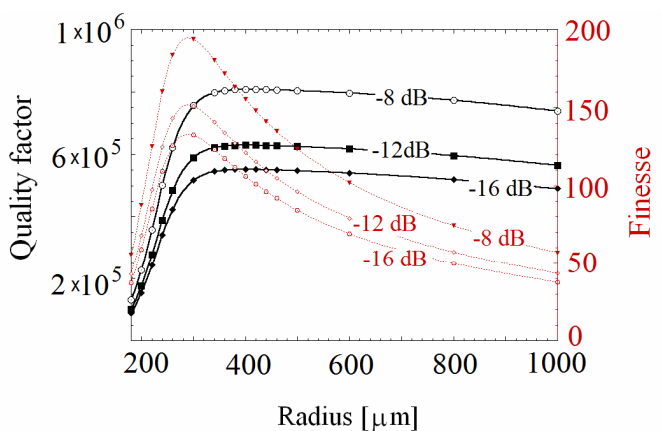

(a)

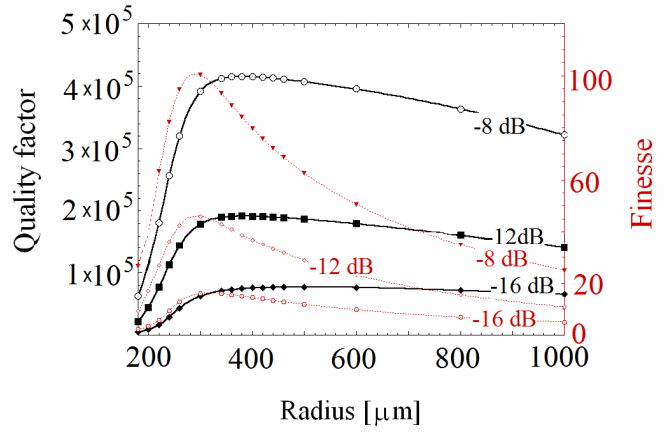

(c)

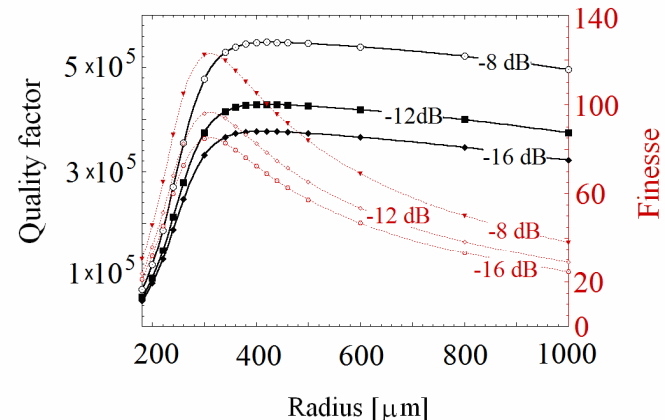

(b)

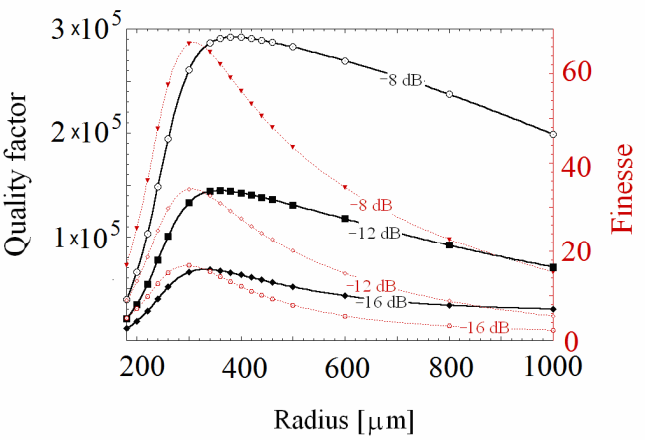

(d)

FIG. 9 (a) Quality factor and finesse dependence on $R$ for one bus waveguide architecture and quasi-TE mode, (b) one bus waveguide architecture and quasi-TM mode, (c) two bus waveguides architecture and quasi-TE mode, (d) two bus waveguides architecture and quasi-TM mode. 


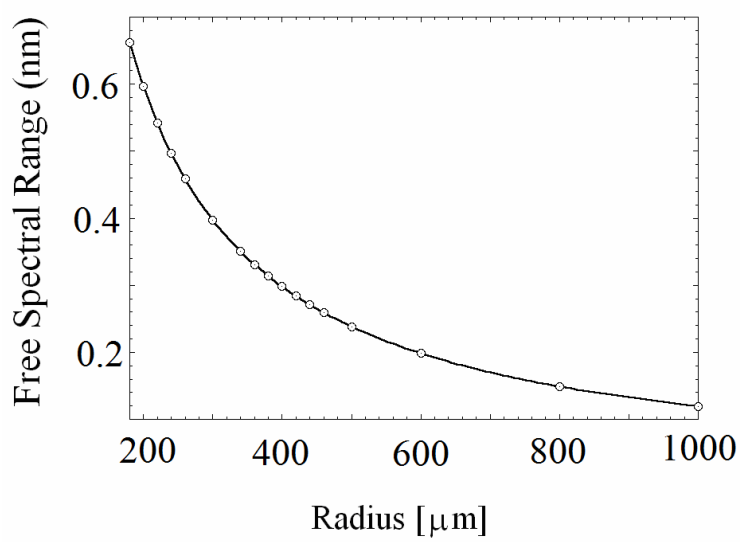

(a)

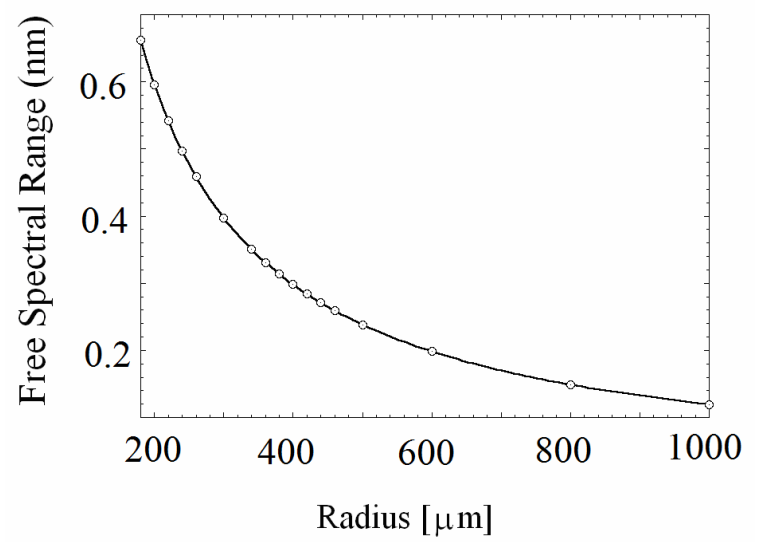

(b)

FIG. 10 (a) FSR dependence on ring resonator radius for quasi-TE and (b) quasi-TM modes.

FSR is not influenced by the device's geometry (one/two bus waveguides) but only by incoming light polarization (this influence is quite modest because the adopted waveguide exhibits a very reduced birefringence). For quasi-TE and quasiTM mode, we can see that FSR monotonically decreases by increasing $R$. Maximum achievable FSR value is lower than $0.7 \mathrm{~nm}$ whereas FSR is equal to $0.3-0.4 \mathrm{~nm}$ for $R$ ranging from $300 \mu \mathrm{m}$ to $400 \mu \mathrm{m}$ (this is the range in which $Q$ and $F$ are maximum).

Optimization procedure results previously shown have been obtained assuming same waveguide as in [35], having a width $w=900 \mathrm{~nm}$, a height $h=400 \mathrm{~nm}$ and an index contrast $\Delta n=$ $n_{1}-n_{2}$ equal to 0.193 . We have also investigated the effect of waveguide width and index contrast change on ring resonator quality factor and finesse. Three index contrast values $(0.282$, 0.193 and 0.159 ) corresponding to three different core materials have been considered. In particular the possibility of using $\mathrm{In}_{0 \cdot 66} \mathrm{Ga}_{0.34} \mathrm{As}_{0.76} \mathrm{P}_{0.24}\left(n_{1}=3.450\right), \mathrm{In}_{0.75} \mathrm{Ga}_{0.25} \mathrm{As}_{0.55} \mathrm{P}_{0.45}$ $\left(n_{1}=3.361\right)$ and $\operatorname{In}_{0.8} \mathrm{Ga}_{0.2} \mathrm{As}_{0.45} \mathrm{P}_{0.55}\left(n_{1}=3.327\right)$ as core materials has been investigated. Lattice constants of all these materials are matched with InP lattice constant. Waveguide width has been varied in the range from $700 \mathrm{~nm}$ to $1100 \mathrm{~nm}$. In this analysis, the ring radius of $200 \mu \mathrm{m}$ has been maintained as a constant.

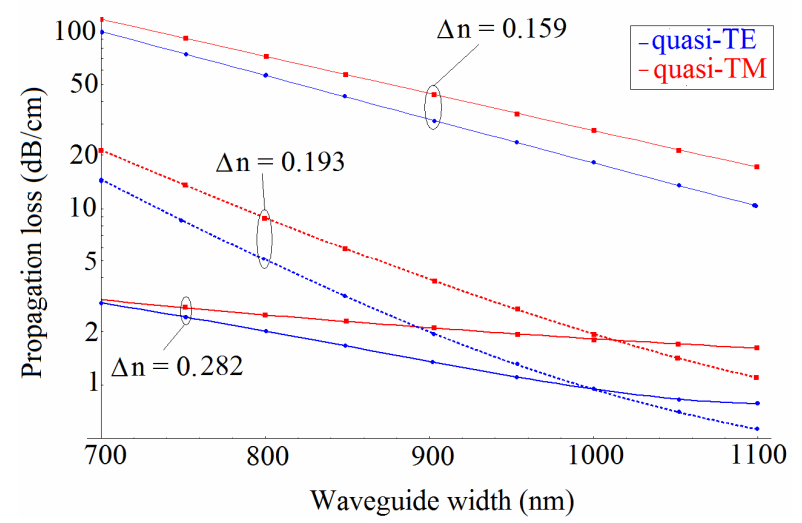

FIG. 11 Propagation loss dependence on waveguide width and $\Delta n(\lambda=1550 \mathrm{~nm}$, $R=200 \mu \mathrm{m})$.

For $R=200 \mu \mathrm{m}$ and both polarizations, propagation loss within the ring has been calculated as a function of $w$ by the method reported in Section 2 (see Figure 11). For all $\Delta n$ values, propagation loss monotonically decreases by increasing $w$, being larger for quasi-TM mode than for quasi-TE one. Minimum value of propagation loss $(\alpha=0.56 \mathrm{~dB} / \mathrm{cm})$ has been achieved for $w=1100 \mathrm{~nm}, \Delta n=0.193$ and quasi-TE mode. Adopting this waveguide features and $R=200 \mu \mathrm{m}$, quality factor values of 434,000 and 286,000 have been obtained for one bus waveguide architecture and two bus waveguides, respectively (in both cases $T_{r j}=-8 \mathrm{~dB}$ ). These $Q$ values are significantly larger than that experimentally obtained in [35] using a resonator having $R=200 \mu \mathrm{m}$ and a non-optimized waveguide.

Main geometrical features and performance parameters of six optimized ring resonator structures are summarized in Table 2. Maximum quality factor $\left(Q=8.08 \times 10^{5}\right)$ is achieved for $R=400 \mu \mathrm{m}, g=1.512 \mu \mathrm{m}$, one bus waveguide architecture, quasi-TE mode and $T_{r j}=-8 \mathrm{~dB}$.

\section{CONCLUSION}

With the purpose of optimizing device performance, quality factor and finesse dependence on the resonator geometry and waveguide, radius, and excited optical mode has been estimated for a $\mathrm{In}_{1-x} \mathrm{Ga}_{x} \mathrm{As}_{y} \mathrm{P}_{1-y} / \mathrm{InP}$ ring resonator employing a buried wave-guiding structure. A very general modelling procedure, adoptable to theoretically investigate and design a wide spectrum of integrated optical ring resonators to be realized in different technologies, has been developed and validated using experimental data in literature.

Reported data show that one I/O bus waveguide architecture permits to achieve larger $Q$ and $F$ values than two bus waveguides one and that the achievement of large $Q$ and $F$ values is conflicting with large through rejections. These two conclusions are quite general and we expect that they are true for different kinds of passive ring resonators.

For one I/O bus architecture maximum quality factor value $\left(8.08 \times 10^{5}\right)$ has been predicted for $R=400 \mu \mathrm{m}$. $\left(T_{r j}=-8 \mathrm{~dB}\right)$. A 32\% decrease in $Q$ value is induced requiring a $T_{r j}$ (in $\mathrm{dB}$ ) 


\begin{tabular}{|c|c|c|c|c|c|c|c|}
\hline $\begin{array}{l}1 \text { BUS / } 2 \\
\text { BUS WGs }\end{array}$ & $\begin{array}{l}\text { Waveguide } \\
\text { features }\end{array}$ & $\begin{array}{c}\text { Radius } \\
(\mu \mathrm{m})\end{array}$ & $g(\mu \mathrm{m})$ & $\begin{array}{l}\text { Quality } \\
\text { Factor }\end{array}$ & Finesse & FSR (nm) & $T_{r j}(\mathrm{~dB})$ \\
\hline 2 & $\begin{array}{l}w=900 \mathrm{~nm} \\
h=400 \mathrm{~nm} \\
\Delta n=0.193\end{array}$ & 380 & 1.318 & 416,000 & 84.1 & 0.31 & -8 \\
\hline 2 & $\begin{array}{c}w=900 \mathrm{~nm} \\
h=400 \mathrm{~nm} \\
\Delta n=0.193\end{array}$ & 500 & 0.759 & 77,000 & 11.8 & 0.24 & -16 \\
\hline 1 & $\begin{array}{c}w=900 \mathrm{~nm} \\
h=400 \mathrm{~nm} \\
\Delta n=0.193\end{array}$ & 400 & 1.512 & 808,000 & 79.8 & 0.3 & -8 \\
\hline 1 & $\begin{array}{c}w=900 \mathrm{~nm} \\
h=400 \mathrm{~nm} \\
\Delta n=0.193\end{array}$ & 400 & 1.346 & 552,000 & 106.1 & 0.3 & -16 \\
\hline 1 & $\begin{array}{c}w=1100 \mathrm{~nm} \\
h=400 \mathrm{~nm} \\
\Delta n=0.193\end{array}$ & 200 & 0.592 & 434,000 & 166.5 & 0.54 & -8 \\
\hline 2 & $\begin{array}{c}w=1100 \mathrm{~nm} \\
h=400 \mathrm{~nm} \\
\Delta n=0.193\end{array}$ & 200 & 0.524 & 286,000 & 109.6 & 0.54 & -8 \\
\hline
\end{tabular}

TABLE 2 Geometrical features and performance parameters of optimized ring resonators structures.

doubling. For two I/O buses architecture, $R$ value maximizing the quality factor is equal to $380 \mu \mathrm{m}$. For this $R$ value, $T_{r j}=-8 \mathrm{~dB}$ and quasi-TE mode, we have $Q=4.16 \times 10^{5}$, $F=84.1$ and FSR $=0.31 \mathrm{~nm}$. Thus, for two bus waveguides architecture, passing from $R=200 \mu \mathrm{m}$ (as in [35]) to $R=380 \mu \mathrm{m}, Q$ becomes quite four times larger and $F$ is doubled at the expense of a FSR reduction of about $20 \%$. Maintaining $R=200 \mu \mathrm{m}$ and optimizing only the waveguide structure, a $Q$ increase of 2.5 times has been proved as obtainable.

\section{ACKNOWLED GEMENTS}

The work has been partially funded by European Space Agency (ESA) under IOLG project n. 1678/02/NL/PA, in the frame of ESA-Politecnico di Bari agreement $n$. 20199/06/NL/PA.

\section{References}

[1] E. A. J. Marcatili, "Bends in Optical Dielectric Guides" AT\&t Tech. J. 48, 2103-2132 (1969).

[2] D. G. Rabus, Integrated Ring Resonators: The Compendium (Springer, Berlin, 2007).

[3] J. Heebner, R. Grover, and T. Irahim, Optical Microresonators: Theory, Fabbrication, and Applications (Springer, Berlin, 2007).

[4] S. Xiao, M. H. Khan, H. Shen, and M. Qi, "Multiple-channel silicon micro-resonator based filters for WDM applications" Opt. Express 15, 7489-7498 (2007).

[5] B. Bortnik, Y.-C. Hung, H. Tazawa, B.-J. Seo, J. Luo, A. K.-Y. Jen, W. H. Steier, and H. R. Fetterman, "Electrooptic Polymer Ring Resonator Modulation up to $165 \mathrm{CHz}$ " IEEE J. Sel. Top. Quant. 13, 104-110 (2007).

[6] K. Suzuki, K. Takiguchi, and K. Hotate, "Monolithically Integrated Resonator Microoptic Cyro on Silica Planar Lightwave Circuit" J. Lightwave Technol. 18, 66-72 (2000).
[7] C. Ciminelli, F. Peluso, and M. N. Armenise, A new integrated optical angular velocity sensor 93-100 (Proceedings SPIE, 5728, 2005).

[8] C. Ciminelli, F. Peluso, E. Armandillo, and M. N. Armenise, Modeling of a new integrated optical angular velocity sensor (Optronics Symposium (OPTRO), Paris, 8 - 12 May 2005).

[9] C. Ciminelli, Innovative photonic technologies for gyroscope systems (EOS Topical Meeting - Photonic Devices in Space, Paris, 18-19 October 2006).

[10] C. Ciminelli, C. E. Campanella, and M. N. Armenise, Design of passive ring resonators to be used for sensing applications $278-280$ (Proc. of First Mediterranean Photonics Conference, 2008).

[11] C. Ciminelli, C. E. Campanella, and M. N. Armenise, "Optimized Design of Integrated Optical Angular Velocity Sensors based on a Passive Ring Resonator" J. Lightwave Technol. (2009).

[12] H. Ma, X. Zhang, Z. Jin, and C. Ding, "Waveguide-type optical passive ring resonator gyro using phase modulation spectroscopy technique" Opt. Eng. 45, 080506 (2006).

[13] C. Vannahme, H. Suche, S. Reza, R. Ricken, V. Quiring, and W. Sohler, Integrated Optical Ti:LiNbO ${ }_{3}$ Ring Resonator for Rotation Rate Sensing WE1 (European Conference on Integrated Optics (ECIO), 2007).

[14] G. Li and K. A. Winick, Integrated optical ring resonators fabricated by silver ion exchange in glass 1-2 (Conference on Lasers and Electro-Optics (CLEO), 1, 2004).

[15] C. Ford, R. Ramberg, K. Johnson, W. Berglund, B. Ellerbusch, R. Schermer, and A. Gopinath, "Cavity Element Resonant Micro Optical Gyroscope" IEEE Aero. El. Sys. Mag. 15, 33-36 (2000).

[16] H. Hsiao and K. A. Winick, "Planar glass waveguide ring resonators with gain" Opt. Express 15, 17783-17797 (2007).

[17] C.-Y. Chao, W. Fung, and L. J. Guo, "Polymer Microring Resonators for Biochemical Sensing Applications" IEEE J. Sel. Topics in Quantum Electron. 12, 134-142 (2006).

[18] A. Yalçin, K. C. Popat, J. C. Aldridge, T. A. Desai, J. Hryniewicz, N. Chbouki, B. E. Little, O. King, V. Van, S. Chu, D. Gill, M. AnthesWashburn, M. S. Unlu, and B. B. Goldberg, "Optical sensing of 
biomelecules using microring resonators" IEEE J. Sel. Top. Quant. 12, 148-155 (2006).

[19] A. Ksendzov and Y. Lin, "Integrated optics ring-resonator sensors for protein detection" Opt. Lett. 30, 3344-3346 (2005).

[20] E. Krioukov, D. J. W. Klunder, A. Driessen, J. Greve, and C. Otto, "Sensor based on an integrated optical cavity" Opt. Lett. 27, 512514 (2002).

[21] B. Bhola and W. H. Steier, "A Novel Optical Microring Resonator Accelerometer" IEEE Sens. J. 7, 1759-1766 (2007).

[22] I. Kiyat, C. Kocabas, and A. Aydinli, "Integrated micro ring resonator displacement sensor for scanning probe Microscopies" J. Micromech. Microeng. 14, 374-381 (2004).

[23] J. Haavisto and G. A. Pajer "Resonance effects in low-loss ring waveguides" Opt. Lett. 12, 510-512 (1980).

[24] R. G. Walker and C. D. W. Wilkinson, "Integrated optical ring resonators made by silver ion-exchange in glass" Appl. Optics 22, 1029-1035 (1983).

[25] R. Adar, M. R. Serbin, and V. Mizrahi, "Less than $1 \mathrm{~dB}$ Per Meter Propagation Loss of Silica Waveguides Measured Using a Ring Resonator" J. Lightwave Technol. 12, 1369-1372 (1994).

[26] C. Ciminelli, F. Dell'olio, C. E. Campanella, V. M. N. Passaro, and M. N. Armenise, "Integrated Optical Ring Resonators: Modelling and Technologies" in Progress in Optical Fibers, P. S. Emersone, ed., (Nova Science Publishers, New York, 2009).

[27] S. Xiao, M. H. Khan, H. Shen, and M. Qi, "Silicon-on-Insulator Microring Add-Drop Filters With Free Spectral Ranges Over $30 \mathrm{nm"} \mathrm{J.}$ Lightwave Technol. 26, 228-236 (2008).

[28] D. G. Rabus, M. Hamacher, U. Troppenz, and H. Heidrich,“HighQ Channel-Dropping Filters Using Ring Resonators With Integrated SOAs" IEEE Photonic. Tech. L. 14, 1442-1444 (2002).

[29] V. M. Menon, W. Tong, and S. R. Forrest, "Control of Quality Factor and Critical Coupling in Microring Resonators Through Integration of a Semiconductor Optical Amplifier" IEEE Photonic. Tech. L. 16, 1343-1345 (2004).

[30] S. J. Choi, Z. Peng, Q. Yang, E. H. Hwang, and P. D. Dapkus, "A HighQ Wavelength Filter Based on Buried Heterostructure Ring Resonators Integrated With a Semiconductor Optical Amplifier" IEEE Photonic. Tech. L. 17, 1210-2103 (2005).

[31] D. Rafizadeh, J. P. Zhang, R. C. Tiberio, and S. T. Ho, "Propagation
Loss Measurements in Semiconductor Microcavity Ring and Disk Resonators" J. Lightwave Technol. 16, 1308-1314 (1998).

[32] P. P. Absil, Microring resonators for wavelength division multiplexing and integrated photonics applications (Ph. D. thesis, University of Maryland, 2000).

[33] D. G. Rabus, and M. Hamacher, "MMI-Coupled Ring Resonators in GalnAsP-InP" IEEE Photonic. Tech. L. 13, 812-814 (2001).

[34] R. Grover, Indium Phosphide Based Optical Micro-Ring Resonators (Ph. D. thesis, University of Maryland, 2003).

[35] S. J. Choi, K. Djordjev, Z. Peng, Q. Yang, S. J. Choi, and P. D. Dapkus, "Laterally Coupled Buried Heterostructure High- $Q$ Ring Resonators" IEEE Photonic. Tech. L. 16, 2266-2268 (2004).

[36] R. Grover, P. P. Absil, T. A. Ibrahim, and P.-T. Ho, “III-V Semiconductor Optical Micro-Ring Resonator" $110-129$ (Proc. of $39^{\text {th }}$ International School of Quantum Electronics, 2003).

[37] F. F. Soares, Photonic integrated true-time-delay beamformers in InP technology (Ph. D. thesis, Technische Universiteit Eindhoven, paragraph 2.3, 2006).

[38] Fimmwave 4.6, Photon Design (2007).

[39] M. Galarza, J. Moreno, M. Lopez-Amo, I. Christiaens, D. Van Thourhout, and R. Baets, "Simple low-loss waveguide bends using ARROW effect" Appl. Phys. B-Lasers 0. 80, 745-748 (2005).

[40] R. Halir, A. Ortega-Monux, J. G. Wangumert-Perez, I. MolinaFernandez, and P. Cheben, "Fabrication Tolerance Analysis of Bent Single-Mode Rib Waveguides on Sol" Opt. Quant. Electron. 38, 921932 (2007).

[41] K. Kakihara, N. Kono, K. Saitoh, and M. Koshiba, "Full-vectorial finite element method in a cylindrical coordinate system for loss analysis of photonic wire bends" Opt. Express 14, 11128-11141 (2006).

[42] C. Ciminelli, V. M. N. Passaro, F. DellâǍźolio, M. N. Armenise, "Three-dimensional modelling of scattering loss in InGaAsP/InP and Silica-on-Silicon bent waveguides" J. Europ. 0pt. Soc. Rap. Public. 4, 09015 (2009).

[43] OptiBPM 9, Optiwave (2007).

[44] A. Yariv, "Universal relations for coupling of optical power between. microresonators and dielectric waveguides" Electron. Lett. $36,321-322(2000)$. 